\title{
Unsteady Hydro-Magnetic Heat and Mass Transfer Flow of a Non-Newtonian Power-Law Fluid past a Flat Plate in the Presence of Homogeneous Chemical Reaction
}

\author{
Itishree Swain', Hadibandhu Pattanayak ${ }^{2}$ \\ ${ }^{1}$ Department of Mathematics, Krupajal Engineering School, Bhubaneswar, India \\ ${ }^{2}$ Department of Mathematics, Institute of Mathematics and Applications, Bhubaneswar, India \\ Email: h.pattnayak@gmail.com
}

Received 11 May 2015; accepted 26 May 2015; published 29 May 2015

Copyright (C) 2015 by authors and Scientific Research Publishing Inc. This work is licensed under the Creative Commons Attribution International License (CC BY). http://creativecommons.org/licenses/by/4.0/

(c) () Open Access

\begin{abstract}
This paper investigates the flow, heat andmass transfer of a power law fluid from a vertical plate in presence of a magnetic field. The resulting non-linear partial differential equations governing the flow together with the boundary conditions are reduced to non-dimensional form. The governing equations are discretized using implicit finite difference scheme and solved numerically. The velocity, temperature and concentration profile are presented graphically while the skin friction, local Nusselt number and the Sherwood number are presented in tabular form for different values of parameters of the problem.
\end{abstract}

\section{Keywords}

Power Law Fluid, Chemical Reaction, Thermal Diffusion, Magnetic Field

\section{Introduction}

The fluids which are encountered in chemical and allied processing applications are known as non-Newtonianfluids. The study of non-Newtonian fluid flows has considerable interest for their numerous engineering applications. During the past four decades the study of non-Newtonian fluids has gained interest because of their numerous technological applications, including manufacturing of the plastic sheets, performance of lubricants and 
movement of biological fluids. To explain the behavior of non-Newtonian fluid different models have been proposed. Among these the power law fluid has gained importance. The order of chemical reactions depends on several factors. One of the simplest chemical reactions is the first order reaction in which the rate of reaction is directly proportional to the species concentration. Now a days, due to the growing use of these non-Newtonian substances in various manufacturing and processing industries, considerable efforts have been directed towards understanding their friction and heat transfer characteristics. By the application of a magnetic field hydromagnetic techniques are used for the purification of molten metal. The problem of steady flow and heat transfer in power law fluid by free convection along a vertical plate has been investigated by many researchers. Vujanovic et al. [1] investigated a variational solution of the Rayleigh problem for a power law non-Newtonian conducting fluid. Padhy \& Pattnayak [2] studied the mass transfer and free convective effects of a power law fluid past an impulsively started vertical plate. Murthy [3] investigated effects of double dispersion on mixed convection heat and mass transfer in a non-Darcy porous medium. Muthucumaraswamy et al. [4] discussed on diffusion and first order chemical reaction on impulsively started infinite vertical plate with variable temperature. Naseer [5] investigated the problem of unsteady free convection with heat $\&$ mass transfer from an isothermal vertical flat plate to a non-Newtonian power law fluid immersed in a saturated porous medium. Chamkha et al. [6] investigated unsteady natural convective power law fluid flow past a vertical plate embedded in a non-Darcian porous medium in the presence of a homogeneous chemical reaction. Khan et al. [7] discussed non-Newtonian MHD mixed convective power law fluid flow over a vertical stretching sheet with thermal radiation, heat generation and chemical reaction effects. Olajuwon et al. [8] studied convection heat mass transfer in a power law fluid with non-constant relaxation time past a vertical porous plate in the presence of thermo and thermal diffusion. Olajuwon [9] examined effects of thermo diffusion and chemical reaction on heat and mass transfer in a power law fluid over a flat plate with heat generation. Then in 2014 many researchers had shown interest to examine on the subject heat and mass transfer in a non-Newtonian fluid. Uwanta et al. [10] investigated heat and mass transfer flow past an infinite vertical plate with variable thermal conductivity heat source and chemical reaction. Jothimani and Vidhya [11] studied non-Newtonian fluid flow and heat transfer over a non-linearly stretching surface along with porous plate in porous medium. Recently Madhu et al. [12] studied effect of viscous dissipation and thermal stratification on chemical reacting fluid flow over a vertical stretching surface with heat source.

The aim of the present work is to investigate the unsteady hydro magnetic non-Newtonian power law fluid past a flat plate with heat and mass transfer effect. The governing equations, describing the model are highly nonlinear coupled partial differential equations in nature. Hence closed form solutions are not possible. Suitable implicit finite difference scheme has been used to get the solution of the problem. Graphs have been plotted against various flow parameters to study the characteristics of velocity, temperature and concentration of the fluid.

\section{Formulation of the Problem}

There exist different types of non-Newtonian fluids but the simplest and most common type is the power-law fluid for which the rheological equation of the state between stress components and strain rate components defined by Vujanovic is

$$
\tau_{i j}=-P \delta_{i j}+K\left|\sum_{m=1}^{3} \sum_{i=0}^{3} e_{i m} e_{i m}\right|^{\frac{n-1}{2}} e_{i j}
$$

where, $P$ is the pressure, $\delta_{i j}$ is the Kronecker delta, $K$ and $n$ are the consistency and flow behavior indices of the fluid respectively. When $n>1$ the fluid is described as dilatant, $n<1$ as pseudo-plastic and when $n=1$ it is known as the Newtonian fluid.

Consider the unsteady free convection heat and mass transfer flow of a two-dimensional, viscous, incompressible, electrically conducting and chemically reactive non-Newtonian power-law fluid along an infinite nonconducting vertical flat plate in the presence of a uniform magnetic field $B_{0}$ applied in a transverse direction to fluid flow. Let $x^{\prime}$-axis be along the plate in upward direction, $y^{\prime}$-axis is normal to it $\& z^{\prime}$-axis is normal to $x^{\prime} y^{\prime}$-plane. Initially, at time $t^{\prime} \leq 0$, the fluid and plate are at rest and at a uniform temperature $T_{\infty}^{\prime}$. When $t^{\prime}>0$ the plate is maintained at constant temperature $T_{\infty}^{\prime}$ and constant species concentration $C_{w}^{\prime}$. Since the plate is of 
infinite extent in $x^{\prime}$ direction and is electrically non-conducting, except pressure all other physical quantities are functions of $y^{\prime}$ and $t^{\prime}$ only. The governing equations describing the model are

$$
\begin{aligned}
& \frac{\partial u^{\prime}}{\partial x^{\prime}}+\frac{\partial v^{\prime}}{\partial y^{\prime}}=0 \\
& \frac{\partial u^{\prime}}{\partial t^{\prime}}+u \frac{\partial u^{\prime}}{\partial x^{\prime}}+v^{\prime} \frac{\partial u^{\prime}}{\partial y^{\prime}}=g\left[\beta_{T}\left(T^{\prime}-T_{\infty}^{\prime}\right)+\beta_{C}\left(C^{\prime}-C_{\infty}^{\prime}\right)\right]+\frac{k \partial}{\rho \partial y^{\prime}}\left|\frac{\partial u^{\prime}}{\partial y^{\prime}}\right|^{n-1} \frac{\partial u^{\prime}}{\partial y^{\prime}}-\frac{\sigma B_{0}^{2} u^{\prime}}{\rho} \\
& \frac{\partial T^{\prime}}{\partial t^{\prime}}+u^{\prime} \frac{\partial T^{\prime}}{\partial x^{\prime}}+v^{\prime} \frac{\partial T^{\prime}}{\partial y^{\prime}}=\alpha \frac{\partial^{2} T^{\prime}}{\partial y^{\prime 2}} \\
& \frac{\partial C^{\prime}}{\partial t^{\prime}}+u^{\prime} \frac{\partial C^{\prime}}{\partial x^{\prime}}+v^{\prime} \frac{\partial C^{\prime}}{\partial y^{\prime}}=D \frac{\partial^{2} C^{\prime}}{\partial y^{\prime 2}}-k_{c}\left(C^{\prime}-C_{\infty}^{\prime}\right)
\end{aligned}
$$

where $g$ is acceleration due to gravity, $\alpha$ represents the thermal diffusivity, $\beta_{T}$ is coefficient of thermal expansion of fluid, $\beta_{C}$ is volumetric coefficient of expansion or contraction, $k$ is thermal conductivity of the fluid, $\rho$ is fluid density, $n$ is power law index, $u^{\prime} \& v^{\prime}$ are stream wise and transverse velocity respectively.

Similarly $x^{\prime}$ and $y^{\prime}$ are stream wise and transverse co-ordinate. $T^{\prime}$ is temperature of the fluid and $t^{\prime}$ is time, $D$ is the coefficient of mass diffusivity, $k_{c}$ is the rate of chemical reaction, $T_{\infty}^{\prime}$ and $C_{\infty}^{\prime}$ are the free stream temperature and concentration of the fluid respectively, and $T_{w}^{\prime}$ and $C_{w}^{\prime}$ are the temperature and concentration at the wall respectively.

The initial and boundary conditions are

$$
\left.\begin{array}{l}
t^{\prime}=0, u^{\prime}=v^{\prime}=0, T^{\prime}=T_{\infty}^{\prime}, C^{\prime}=C_{\infty}^{\prime}, \forall x^{\prime} \text { and } y^{\prime} \\
t^{\prime}>0, x^{\prime}=0, y^{\prime}=0, T^{\prime}=T_{\infty}^{\prime}, C^{\prime}=C_{\infty}^{\prime} \\
t^{\prime}>0, u^{\prime}=v^{\prime}=0, T^{\prime}=T_{w}^{\prime}, C^{\prime}=C_{w}^{\prime}, \text { at } y^{\prime}=0, x^{\prime}>0 \\
t^{\prime}>0, u^{\prime}=0, T^{\prime}=T_{\infty}^{\prime}, C^{\prime}=C_{\infty}^{\prime} \text { at } y^{\prime} \rightarrow \infty, x^{\prime}>0
\end{array}\right\}
$$

The dimensionless variables are defined as follows:

$$
x=\frac{x^{\prime}}{l}, y=\frac{y^{\prime}}{l}, u=\frac{u^{\prime}}{U_{0}}, v=\frac{v^{\prime}}{U_{0}}, T=\frac{T^{\prime}-T_{\infty}^{\prime}}{T_{w}^{\prime}-T_{\infty}^{\prime}}, C=\frac{C^{\prime}-C_{\infty}^{\prime}}{C_{w}^{\prime}-C_{\infty}^{\prime}}, t=\frac{U_{0} t^{\prime}}{l}
$$

where, $U_{0}=\left[\frac{\rho l^{n}}{k}\right]^{\frac{1}{n-2}}$ and $l$ is the suitable length scale. Substituting the above non-dimensional variables into Equations (2)-(5) yield the following dimensionless equations

$$
\begin{aligned}
& \frac{\partial u}{\partial x}+\frac{\partial v}{\partial y}=0 \\
& \frac{\partial u}{\partial t}+u \frac{\partial u}{\partial x}+v \frac{\partial u}{\partial y}=\frac{\partial}{\partial y}\left|\frac{\partial u}{\partial y}\right|^{n-1} \frac{\partial u}{\partial y}+G_{r} T+G_{m} C-M u \\
& \frac{\partial T}{\partial t}+u \frac{\partial T}{\partial x}+v \frac{\partial T}{\partial y}=\frac{1}{R_{e} P_{r}} \frac{\partial^{2} T}{\partial y^{2}} \\
& \frac{\partial C}{\partial t}+u \frac{\partial C}{\partial x}+v \frac{\partial C}{\partial y}=\frac{1}{R_{e} S_{C}} \frac{\partial^{2} C}{\partial y^{2}}-K_{r} C
\end{aligned}
$$

where $R_{e}=\frac{U_{0} l}{v}$ is the Reynold number, $G_{r}=\frac{g \beta_{T}\left(T_{w}^{\prime}-T_{\infty}^{\prime}\right) l}{U_{0}^{2}}$ is the Grashof number, $G_{m}=\frac{g \beta_{C}\left(C_{w}^{\prime}-C_{\infty}^{\prime}\right) l}{U_{0}^{2}}$ is the modified Grashof number, $P_{r}=\frac{\rho v C_{p}}{k}$ is the Prandtl number, $S_{C}=\frac{v}{D}$ is the Schmidt number, 
$M=\frac{\sigma B_{0}^{2} l}{U_{0} \rho}$ is the magnetic parameter, $K_{r}=\frac{k_{c} l}{U_{0}}$ is the chemical reaction parameter, $C_{p}$ is the specific heat at constant pressure, $v=\frac{\mu}{\rho}$ is the kinematic viscosity and $\mu$ is the constant viscosity of the fluid in boundary layer region.

Accordingly, the initial and boundary conditions will be reduced to

$$
\left.\begin{array}{l}
t=0, u=v=0, T=0, C=0, \forall x \text { and } y \\
t>0, u=v=0, T=C=0, \text { at } x=0, \\
t>0, u=v=0, T=1, C=1 \text { at } y=0, x>0 \\
t>0, u=0, T=0, C=0 \text { at } y \rightarrow \infty, x>0
\end{array}\right\}
$$

The special significance of this type of flow with heat and mass transfer situation are the skin-friction coefficient $C_{\text {f }}$, the local Nusselt number $N_{u}$ and Sherwood number $S_{h}$. These physical quantities are defined in nondimensional form, respectively, as follows:

$$
\begin{gathered}
C_{f} R_{e}^{\frac{1}{2}}=\left|\frac{\partial u}{\partial y}\right|_{y=0}^{n} \\
N_{u} R_{e}^{-\frac{1}{2}}=-\left[\frac{\partial T}{\partial y}\right]_{y=0} \\
S_{h} R_{e}^{-\frac{1}{2}}=-\left[\frac{\partial C}{\partial y}\right]_{y=0} .
\end{gathered}
$$

\section{Solution of the Problem}

The Equations (7)-(10) are solved by implicit finite difference method. For discretization in space and time a uniform mesh of step $\Delta x$ and $\Delta y$ along $x \& y$ direction respectively and time $\Delta t$ are employed so that the grid points are $\left(x_{i}, y_{j}, t_{k}\right)=(i \Delta x, j \Delta y, k \Delta t)$ for $i=1,2, \cdots, N, j=1,2, \cdots, M \& k=0,1,2, \cdots, K$. The discretized form of Equation (7), (8), (9) and (10) are obtained respectively as,

$$
\begin{gathered}
\quad \frac{u_{i, j}^{k+1}-u_{i-1, j}^{k+1}}{\Delta x}+\frac{v_{i, j}^{k+1}-v_{i, j-1}^{k+1}}{\Delta y}=0 \\
\frac{u_{i, j}^{k+1}-u_{i, j}^{k}}{\Delta t}+u_{i, j}^{k}\left(\frac{u_{i, j}^{k}-u_{i-1, j}^{k}}{\Delta x}\right)+v_{i, j}^{k}\left(\frac{u_{i, j+1}^{k}-u_{i, j}^{k}}{\Delta y}\right) \\
=G_{r} T_{i, j}^{k+1}+G_{m} C_{i, j}^{k+1}-M u_{i, j}^{k+1}+\frac{1}{\Delta y}\left[\left|\frac{u_{i, j+1}^{k}-u_{i, j}^{k}}{\Delta y}\right|^{n-1}\left(\frac{u_{i, j+1}^{k}-u_{i, j}^{k}}{\Delta y}\right)\right]-\frac{1}{\Delta y}\left[\left|\frac{u_{i, j}^{k}-u_{i, j-1}^{k}}{\Delta y}\right|^{n-1}\left(\frac{u_{i, j}^{k}-u_{i, j-1}^{k}}{\Delta y}\right)\right] \\
{\left[\frac{T_{i, j}^{k+1}-T_{i, j}^{k}}{\Delta t}+u_{i, j}^{k}\left(\frac{T_{i, j}^{k}-T_{i-1, j}^{k}}{\Delta x}\right)+v_{i, j}^{k}\left(\frac{T_{i, j+1}^{k}-T_{i, j}^{k}}{\Delta y}\right)\right]=\frac{1}{P_{r} R_{e}}\left[\frac{T_{i, j+1}^{k}-2 T_{i, j}^{k}+T_{i, j-1}^{k}}{(\Delta y)^{2}}\right] .} \\
\frac{C_{i, j}^{k+1}-C_{i, j}^{k}}{\Delta t}+u_{i, j}^{k}\left(\frac{C_{i, j}^{k}-C_{i-1, j}^{k}}{\Delta x}\right)+v_{i, j}^{k}\left(\frac{C_{i, j+1}^{k}-C_{i, j}^{k}}{\Delta y}\right)=\frac{1}{R_{e} S_{c}}\left[\frac{C_{i, j+1}^{k}-2 C_{i, j}^{k}+C_{i, j-1}^{k}}{(\Delta y)^{2}}\right]-K_{r} C_{i, j}^{k+1} .
\end{gathered}
$$

The above discretized Equations (15)-(18) are solved iteratively using the following algorithm.

Step I

Initialize $u_{i, j}^{(0)}, v_{i, j}^{(0)}, T_{i, j}^{(0)}, C_{i, j}^{(0)} \forall(i, j)$.

Step II 
For $k=0,1,2,3, \cdots, k$ max

For $i=1,2, \cdots, N$

For $j=1,2, \cdots, M$

$$
\begin{aligned}
C_{i, j}^{k+1}= & \frac{C_{i, j}^{k}}{1+K_{r} \Delta t}+\frac{\Delta t}{R_{e} S_{c}\left(1+K_{r} \Delta t\right)}\left(\frac{C_{i, j+1}^{k}-2 C_{i, j}^{k}+C_{i, j-1}^{k}}{(\Delta y)^{2}}\right) \\
& -\frac{\Delta t}{1+K_{r} \Delta t}\left[u_{i, j}^{k}\left(\frac{C_{i, j}^{k}-C_{i-1, j}^{k}}{\Delta x}\right)+v_{i, j}^{k}\left(\frac{C_{i, j+1}^{k}-C_{i, j}^{k}}{\Delta y}\right)\right] .
\end{aligned}
$$

Step III

$$
T_{i, j}^{k+1}=T_{i, j}^{k}+\frac{\Delta t}{P_{r} R_{e}}\left(\frac{T_{i, j+1}^{k}-2 T_{i, j}^{k}+T_{i, j-1}^{k}}{\Delta y^{2}}\right)-\Delta t\left[u_{i, j}^{k}\left(\frac{T_{i, j}^{k}-T_{i-1, j}^{k}}{\Delta x}\right)+v_{i, j}^{k}\left(\frac{T_{i, j+1}^{k}-T_{i, j}^{k}}{\Delta y}\right)\right] .
$$

Step IV

$$
\begin{aligned}
u_{i, j}^{k+1}= & \frac{u_{i, j}^{k}}{(1+M \Delta t)}+\frac{\Delta t}{(1+M \Delta t)} G_{r} T_{i, j}^{k+1}+\frac{\Delta t}{(1+M \Delta t)} G_{m} C_{i, j}^{k+1} \\
& +\frac{\Delta t}{(1+M \Delta t) \Delta y}\left[\left|\frac{u_{i, j+1}^{k}-u_{i, j}^{k}}{\Delta y}\right|^{n-1}\left(\frac{u_{i, j+1}^{k}-u_{i, j}^{k}}{\Delta y}\right)\right] \\
& -\frac{\Delta t}{(1+M \Delta t) \Delta y}\left[\left|\frac{u_{i, j}^{k}-u_{i, j-1}^{k}}{\Delta y}\right|^{n-1}\left(\frac{u_{i, j}^{k}-u_{i, j-1}^{k}}{\Delta y}\right)\right] \\
& -\frac{\Delta t}{(1+M \Delta t)}\left[u_{i, j}^{k}\left(\frac{u_{i, j}^{k}-u_{i-1, j}^{k}}{\Delta x}\right)+v_{i, j}^{k}\left(\frac{u_{i, j+1}^{k}-u_{i, j}^{k}}{\Delta y}\right)\right] .
\end{aligned}
$$

Step V

$$
v_{i, j}^{k+1}=\Delta y\left(\frac{u_{i, j}^{k+1}-u_{i-1, j}^{k+1}}{\Delta x}\right) .
$$

The Steps (II)-(V) are repeated until the relative errors of two consecutive values of $u_{i, j}^{k}, v_{i, j}^{k}, T_{i, j}^{k}, C_{i, j}^{k}$ are less than a given tolerance.

\section{Results \& Discussion}

The non-linear governing Equations (7)-(10) with the boundary conditions (11) are solved using finite difference method. The velocity, temperature, and concentration of the fluid for different Reynold numbers are shown in Figures 1(a)-(c). The velocity, temperature and concentration decrease as $R_{e}$ increases.

The velocity and temperature of the fluid for different Prandtl numbers are shown in Figure 2(a) and Figure 2(b). Prandtl number increases the viscous diffusivity of the fluid at the surface which enhanced the velocity of the fluid near the surface as depicted in Figure 2(a), increase in $P_{r}$ implies flow of liquid with low thermal diffusivity and high viscous stress, which increases thermal boundary layer thickness near the surface as shown in Figure 2(b).

In Figure 3(a) and Figure 3(b) we have seen as chemical reaction parameter increases the velocity and species concentration decrease, but it is reverse in the case of Schmidt number. The velocity and species concentration increase as $S_{c}$ increases it is reflected through Figure 4(a) and Figure 4(b).

It is evident from Figure 5 that the presence of transverse magnetic field has a retarding effect on velocity field. But from Figures 6-8 it is observed that with an increase in $G_{r}, G_{m}$ or $N$ the velocity increases.

For the physical interest in view we found the influence of power law index, magnetic parameter, Prandtl number, Reynold number, Schmidt number, chemical reaction parameter, thermal Grashof number and modified 


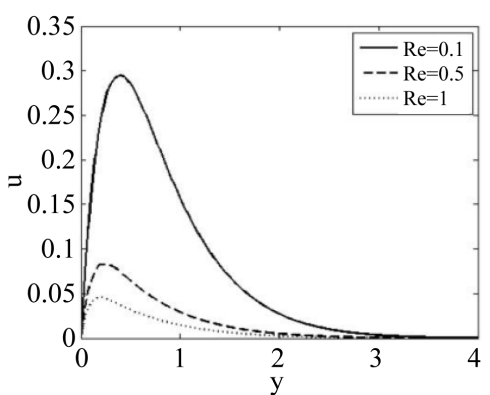

(a)

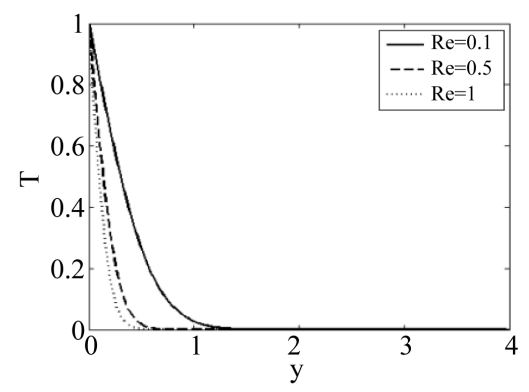

(b)

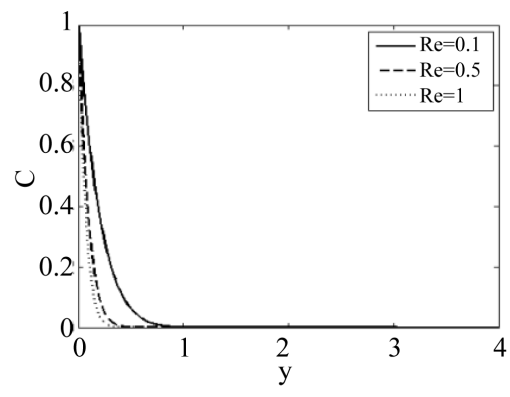

(c)

Figure 1. Variation of $R_{e}$ on (a) fluid velocity, (b) temperature and (c) species concentration when $N=1 ; G_{r}=5 ; G_{m}=5 ; M$ $=1 ; P_{r}=1 ; S_{c}=0.5 ; K_{r}=1$.

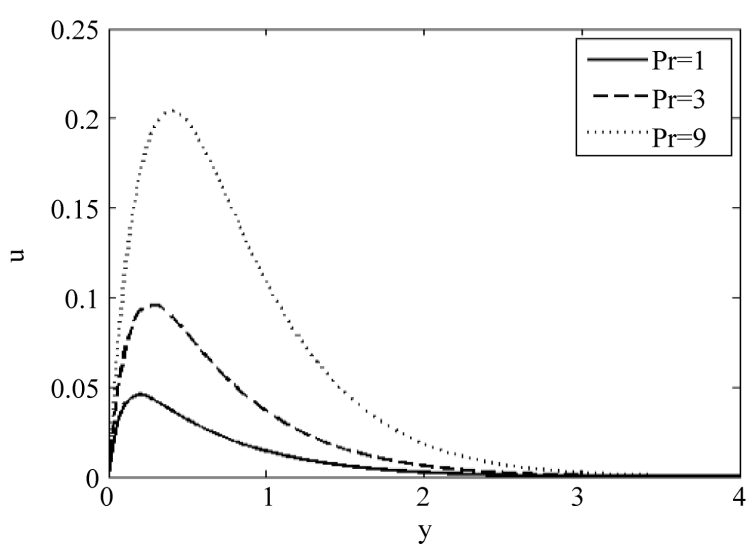

(a)

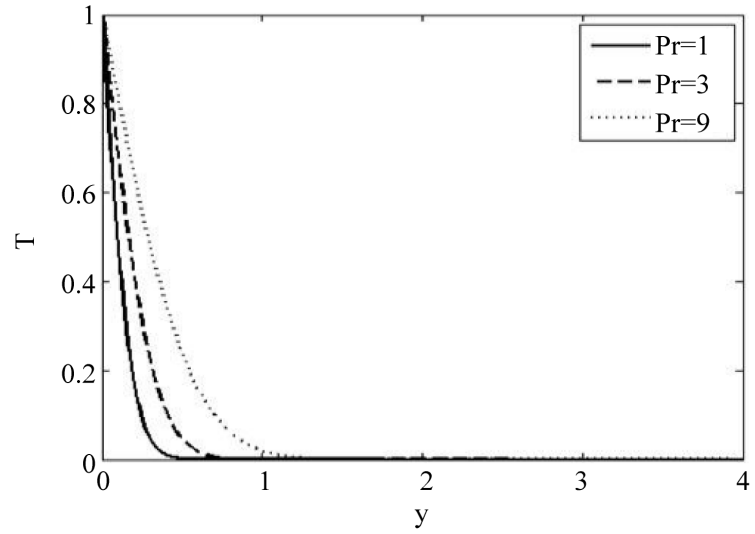

(b)

Figure 2. Variation of $P_{r}$ on (a) fluid velocity and (b) temperature when $N=1 ; G_{r}=5 ; G_{m}=5 ; M=1 ; R_{e}=1 ; S_{c}=0.5 ; K_{r}=$ 1.

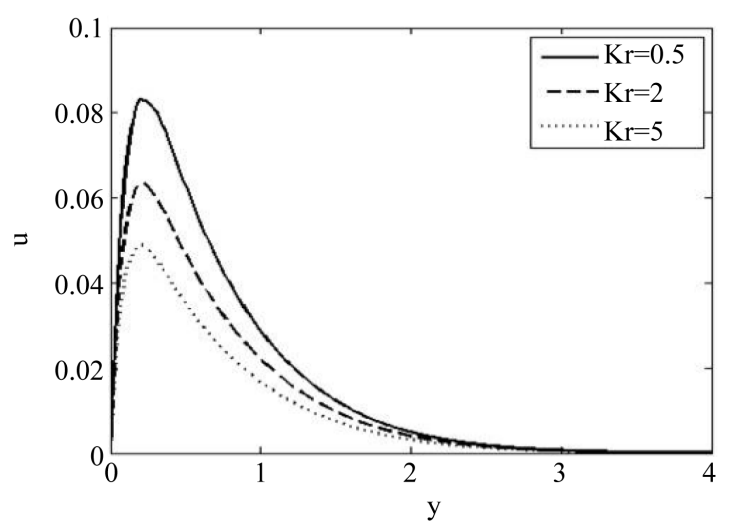

(a)

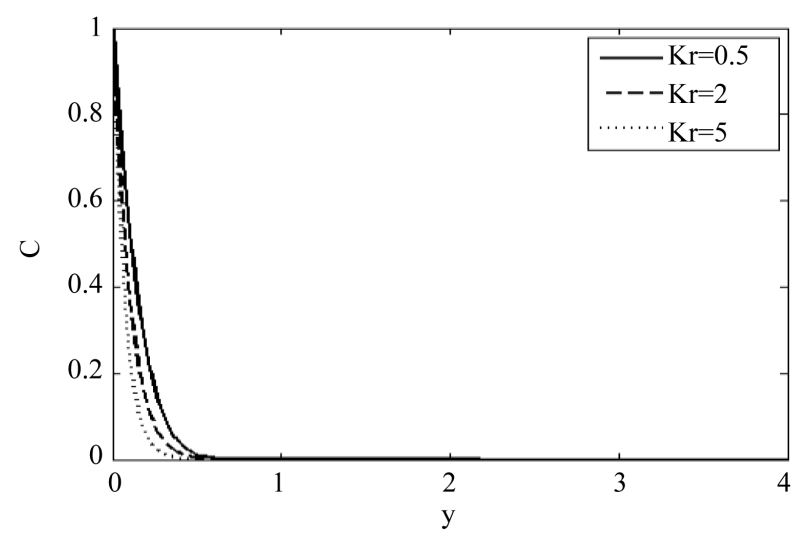

(b)

Figure 3. Variation of $K_{r}$ on (a) Velocity and (b) species concentration when $N=1 ; G_{r}=5 ; G_{m}=5 ; M=1 ; R_{e}=1 ; P_{r}=1 ; S_{c}$ $=2$.

Grashof number on the skin friction $C_{f}$, local Nusselt number $N_{u}$ and Sherwood number $S_{h}$ is shown in the Table 1. It is interesting to note that the increase in magnetic parameter decreases the velocity of the fluid that helps to reduce the skin friction at the surface, but the local heat transfer and mass transfer are not influenced by the magnetic parameter. When the fluid is dilatant the skin friction at the surface decreases, but local Nusselt number does not change. As Prandtl number increases the skin friction increases, local Nusselt number decreases but 


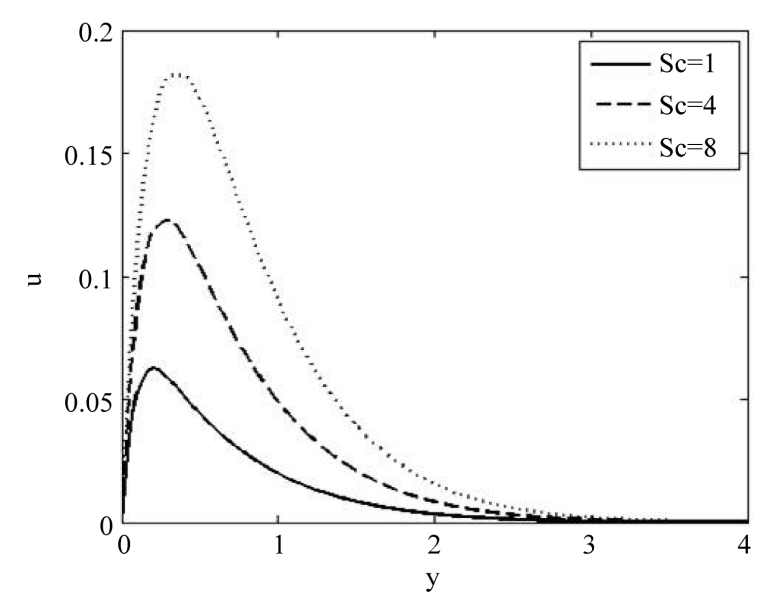

(a)

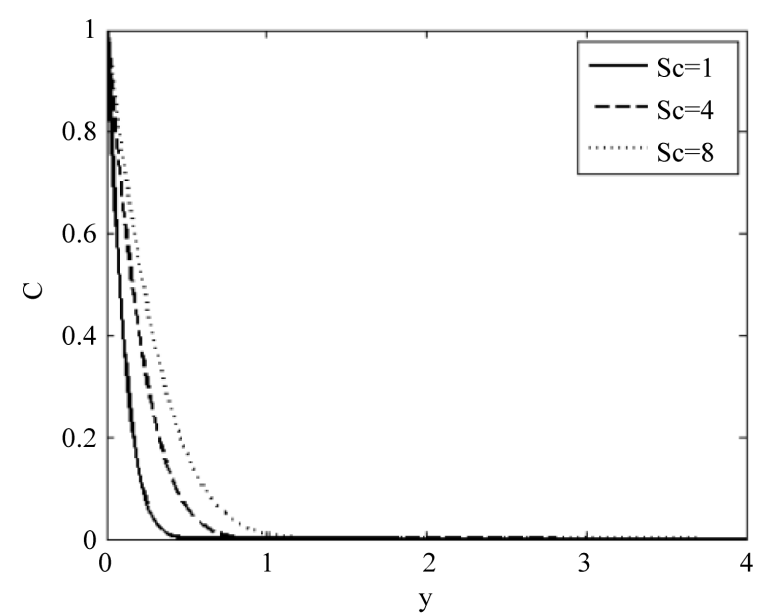

(b)

Figure 4. Variation of $S_{c}$ on (a) fluid velocity and (b) species concentration when $N=1 ; G_{r}=5 ; G_{m}=5 ; M=1 ; R_{e}=1 ; P_{r}=$ $1 ; K_{r}=0.4$.

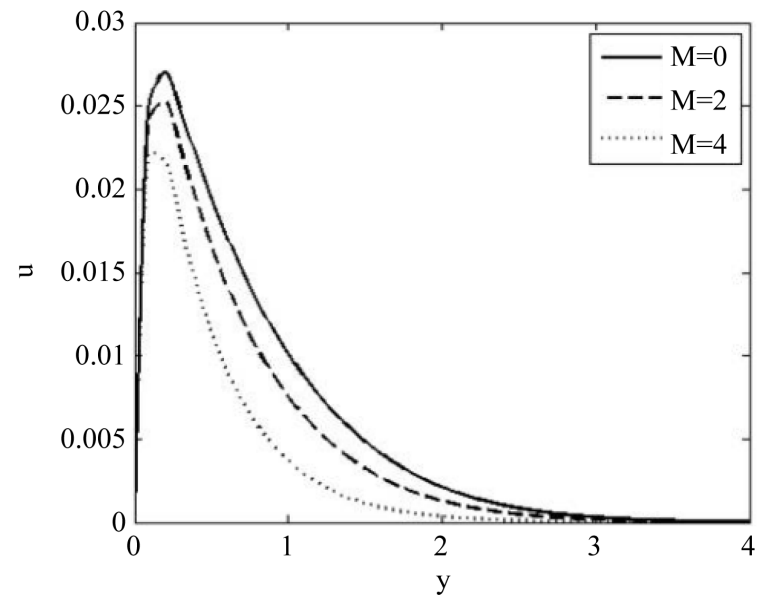

Figure 5. Variation of $M$ on velocity when $N=1 ; G_{r}=5$; $G_{m}=5 ; P_{r}=1 ; S_{c}=0.6 ; K_{r}=1$.

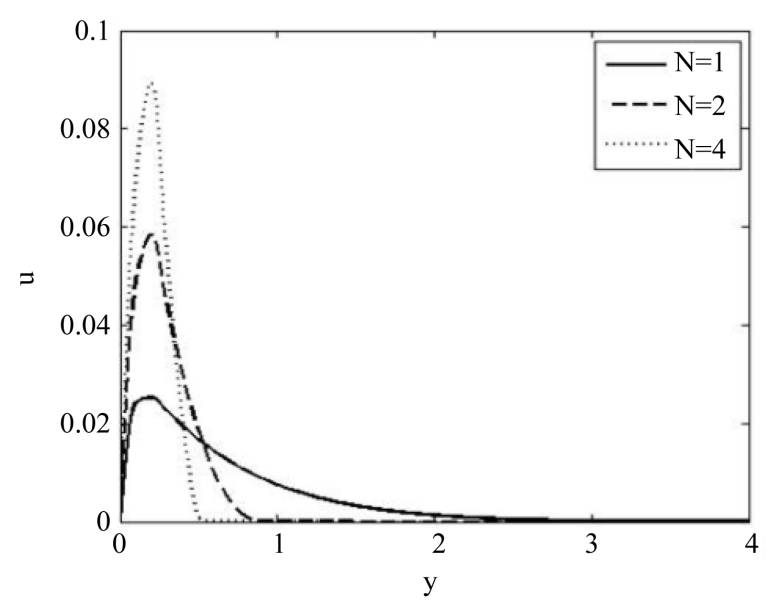

Figure 6. Variation of $N$ on velocity when $G_{r}=5, G_{m}=5$, $M=1, R_{e}=2, P_{r}=1, S_{c}=0.6, K_{r}=1$. 


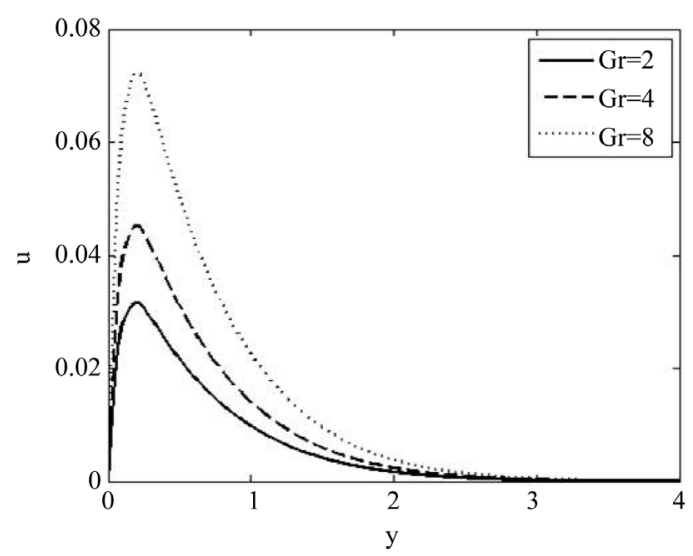

Figure 7. Variation of $G_{r}$ on velocity when $N=1, G_{m}=$ $5, M=1, R_{e}=1, P_{r}=1, S_{c}=0.6, K_{r}=0.4$.

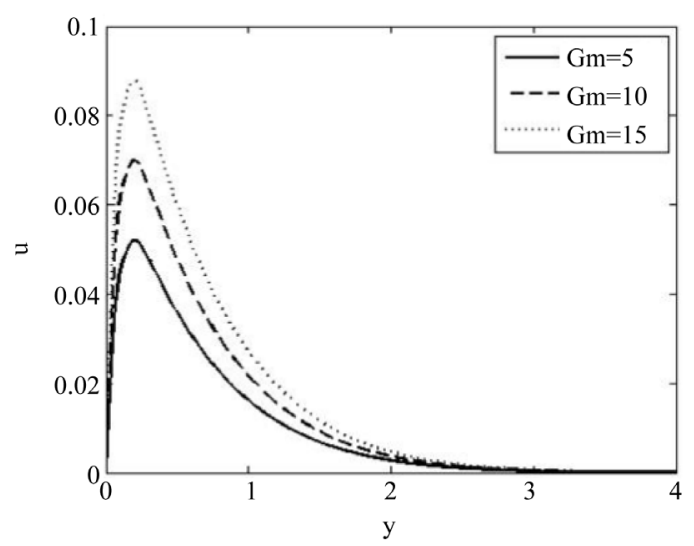

Figure 8. Variation of $G_{m}$ on velocity when $N=1 ; G_{r}=$ 5; $M=1 ; R_{e}=1 ; P_{r}=1 ; S_{c}=0.6 ; K_{r}=0.4$.

Table 1. Skin friction, Nusselt number and Sherwood number.

\begin{tabular}{|c|c|c|c|c|c|c|c|c|c|c|}
\hline$n$ & M & $P_{r}$ & $R_{e}$ & $S_{c}$ & $K_{r}$ & $G_{r}$ & $G_{m}$ & $C_{f}$ & $N_{u}$ & $S_{h}$ \\
\hline 1.5 & & & & & & & & 0.4049 & - & - \\
\hline 2 & 2 & 1 & 1 & 0.6 & 1 & 5 & 5 & 0.4046 & - & - \\
\hline 4 & & & & & & & & 0.4029 & - & - \\
\hline \multirow{2}{*}{-} & 4 & \multirow{2}{*}{-} & \multirow[b]{2}{*}{-} & \multirow[b]{2}{*}{ - } & \multirow[b]{2}{*}{-} & \multirow[b]{2}{*}{-} & \multirow[b]{2}{*}{ - } & 0.3692 & - & - \\
\hline & 8 & & & & & & & 0.3222 & - & - \\
\hline \multirow{2}{*}{-} & \multirow{2}{*}{-} & 2 & \multirow{2}{*}{-} & \multirow{2}{*}{-} & \multirow{2}{*}{-} & \multirow{2}{*}{ - } & \multirow{2}{*}{ - } & 0.5662 & 3.8563 & - \\
\hline & & 4 & & & & & & 0.7651 & 2.7748 & - \\
\hline \multirow{2}{*}{-} & \multirow{2}{*}{-} & \multirow{2}{*}{-} & 3 & \multirow{2}{*}{-} & \multirow{2}{*}{-} & \multirow{2}{*}{-} & \multirow{2}{*}{-} & 0.1658 & 7.5284 & 8.9185 \\
\hline & & & 6 & & & & & 0.0868 & 8.5764 & 9.4168 \\
\hline \multirow{2}{*}{-} & \multirow[b]{2}{*}{-} & \multirow{2}{*}{ - } & \multirow{2}{*}{-} & 1.0 & \multirow[b]{2}{*}{ - } & \multirow{2}{*}{-} & \multirow[b]{2}{*}{ - } & 0.4709 & - & 6.6021 \\
\hline & & & & 1.5 & & & & 0.5386 & - & 5.8548 \\
\hline \multirow{2}{*}{-} & \multirow{2}{*}{-} & \multirow{2}{*}{ - } & \multirow{2}{*}{-} & \multirow{2}{*}{ - } & 2 & \multirow{2}{*}{ - } & \multirow{2}{*}{ - } & 0.3659 & - & 8.1591 \\
\hline & & & & & 3 & & & 0.3425 & - & 8.5691 \\
\hline & & & & & & 10 & - & 0.7066 & 5.2366 & - \\
\hline- & - & - & - & - & - & 15 & & 1.0146 & 5.2366 & - \\
\hline - & - & - & - & - & - & 5 & 10 & 0.5428 & - & 7.4954 \\
\hline- & - & - & - & - & - & 3 & 20 & 0.8224 & - & 7.4954 \\
\hline
\end{tabular}

$C_{f}=$ Skin Friction : $\left(\frac{\partial u}{\partial y}\right)^{n-1} ; N_{u}=$ Local Nusselt Number: $\left(-\frac{\partial T}{\partial y}\right)_{y=0} ; S_{h}=$ Local Nusselt Number: $\left(-\frac{\partial C}{\partial y}\right)_{y=0}$. 
concentration does not change with it. It is evident from the table that with an increase in $R_{e}$ the skin friction decreases while a reverse effect is seen in case of Nusselt number $N_{u}$ and Sherwood number $S_{h}$ is very much affected by Reynold number.

\section{Conclusions}

Unsteady free convective heat and mass transfer in the flow of a two dimensional viscous incompressible electrically conducting and chemically reactive non-Newtonian power-law fluid along an infinite non-conducting vertical flat plate in the presence of uniform magnetic field are studied. It is found that,

- With an increase in $R_{e}$ velocity, temperature \& concentration of the fluid decrease.

- With the increasing value of chemical reaction parameter fluid velocity \& concentration decrease near the plate, but the species concentration shows reverse characteristics as depicted in the skin friction table.

- Magnetic field has a retarding effect on the fluid flow while the thermal radiation has a reverse effect on it. The solutions obtained are well agreed with the Newtonian case and they give improved results, taking into consideration of the behaviour of the magnetic field. This method well suits for other non-Newtonian fluid flow problems.

\section{References}

[1] Vujanovic, B., Stauss, A.M. and Djukic, D. J. (1972) A Variational Solution of the Rayleigh Problem for a Power Law Non-Newtonian Conducting Fluid. Ingenieur-Archiv, 41, 381-386. http://dx.doi.org/10.1007/BF00533141

[2] Padhy, S. and Pattnayak, H.B. (1997) The Finite Difference Solution of Mass Transfer \& Free Convective Effects of a Power Law Fluid past an Impulsively Started Vertical Plate. Journal of OMS, 16, 123-135.

[3] Murthy, P.V.S.N. (2000) Double Dispersion on Mixed Convection Heat \& Mass Transfer in a Non-Darcy Porous Medium. Journal of Heat Transfer, 122, 476-484. http://dx.doi.org/10.1115/1.1286995

[4] Muthucumarswamy, R. and Ganesan, P. (2002) Diffusion \& First Order Chemical Reaction on Impulsively Started Infinite Vertical Plate with Variable Temperature. Journal of Thermal Science, 41, 475-479. http://dx.doi.org/10.1016/S1290-0729(02)01340-6

[5] Elgazery, N. (2008) Transient Analysis of Heat and Mass Transfer by Natural Convection in Power-Law Fluid past a Vertical Plate Immersed in a Porous Medium. Journal of Application \& Applied Mathematics, 3, 267-285.

[6] Chamka, A.J., Aly, A.M. and Mansur, M.A. (2010) Unsteady Natural Convective Power Law Fluid Flow past a Vertical Plate Embedded in a Non-Darcian Porous Medium in the Presence of a Homogeneous Chemical Reaction. Journal of Non-Linear Analysis Modeling \& Control, 15, 139-154.

[7] Khan, Md.S., Karim, I. and Biswas, Md.H.A. (2012) Non-Newtonian MHD Mixed Convective Power Law Fluid Flow over a Vertical Stretching Sheet with Thermal Radiation, Heat Generation \& Chemical Reaction Effect. Journal of Part-1: Natural and Applied Science, 3.

[8] Olajuwan, B.I. and Oyelakin, I.S. (2012) Convection Heat \& Mass Transfer in a Power Law Fluid with Non-Constant Relaxation Time past a Vertical Porous Plate in the Presence of Thermo and Thermal Diffusion. Journal of Theoretical and Applied Mathematics, 42, 53-74.

[9] Olajuwan, B.I. (2013) Effect of Thermo Diffusion and Chemical Reaction on Heat and Mass Transfer in a Power Law Fluid over a Flat Plate with Heat Generation. International Journal of Non-Linear Science, 15, 117-127.

[10] Uwanta, I.J. and Sani, M. (2014) Heat and Mass Transfer Flow past an Infinite Vertical Plate with Variable Thermal Conductivity, Heat Source and Chemical Reaction. The International Journal of Engineering and Science, 3, 77-89.

[11] Jothimani, S. and Vidhya, T. (2014) Non-Newtonian Fluid Flow and Heat Transfer over a Non-Linearly Stretching Surface along with Porous Plate in Porous Medium. International Journal of Engineering Research and Applications, 4, 5-13.

[12] Venkata Madhu, J., Rajsekhar, M.N. and Reddy, B.S. (2015) Effects of Viscous Dissipation and Thermal Stratification on Chemical Reacting Fluid Flow over a Vertical Stretching Surface with Heat Source. Pelagia Research Library Advances in Applied Science Research, 6, 59-65. 